\title{
Formal and informal prediction of recurrent stroke and myocardial infarction after stroke: a systematic review and evaluation of clinical prediction models in a new cohort
}

\author{
Douglas D Thompson ${ }^{1 *}$, Gordon D Murray ${ }^{1}$, Martin Dennis ${ }^{2}$, Cathie LM Sudlow ${ }^{2}$ and William N Whiteley ${ }^{1,2}$
}

\begin{abstract}
Background: The objective of this study was to: (1) systematically review the reporting and methods used in the development of clinical prediction models for recurrent stroke or myocardial infarction (MI) after ischemic stroke; (2) to meta-analyze their external performance; and (3) to compare clinical prediction models to informal clinicians' prediction in the Edinburgh Stroke Study (ESS).

Methods: We searched Medline, EMBASE, reference lists and forward citations of relevant articles from 1980 to 19 April 2013. We included articles which developed multivariable clinical prediction models for the prediction of recurrent stroke and/or Ml following ischemic stroke. We extracted information to assess aspects of model development as well as metrics of performance to determine predictive ability. Model quality was assessed against a pre-defined set of criteria. We used random-effects meta-analysis to pool performance metrics.

Results: We identified twelve model development studies and eleven evaluation studies. Investigators often did not report effective sample size, regression coefficients, handling of missing data; typically categorized continuous predictors; and used data dependent methods to build models. A meta-analysis of the area under the receiver operating characteristic curve (AUROCC) was possible for the Essen Stroke Risk Score (ESRS) and for the Stroke Prognosis Instrument II (SPI-II); the pooled AUROCCs were 0.60 ( $95 \%$ Cl 0.59 to 0.62 ) and 0.62 ( $95 \%$ Cl 0.60 to 0.64), respectively. An evaluation among minor stroke patients in the ESS demonstrated that clinicians discriminated poorly between those with and those without recurrent events and that this was similar to clinical prediction models.
\end{abstract}

Conclusions: The available models for recurrent stroke discriminate poorly between patients with and without a recurrent stroke or $\mathrm{Ml}$ after stroke. Models had a similar discrimination to informal clinicians' predictions. Formal prediction may be improved by addressing commonly encountered methodological problems.

Keywords: Systematic review, Meta-analysis, Stroke, Prediction, Statistical modelling, Evaluation, Development

\section{Background}

About a quarter of the patients who survive their stroke have a recurrent stroke within five years [1]. Any method that could reliably discriminate between those patients at high risk and those at low risk of recurrent stroke would be useful. Patients and their clinicians might use such

\footnotetext{
* Correspondence: D.Thompson-3@sms.ed.ac.uk

'Edinburgh MRC Hub for Trials Methodology Research, Centre for Population Health Sciences, University of Edinburgh Medical School, Teviot Place, Edinburgh EH8 9AG, UK

Full list of author information is available at the end of the article
}

information to make decisions about different preventive strategies and better target resources.

Clinical prediction models (also known as prognostic/ statistical models or scores) combine multiple risk factors to estimate the absolute risk of a future clinical event. No estimate is perfect, but a model that predicted the risk of recurrent stroke just as well as or better than an experienced clinician might improve clinical practice. Some prediction models are used widely in clinical practice to quantify risk of future vascular events (for example, the ASSIGN [2], Framingham [3], and CHADS [4] scores). 
None of the prediction models for recurrent events after stroke is in widespread use, either because their statistical performance is too poor or because the models are too hard to use.

We sought to pool measures of statistical performance of existing models and investigate whether there were aspects of study design or analysis that might be improved in the development of new models. Therefore, we systematically reviewed the literature on the development and evaluation of prediction models for recurrent vascular events after ischemic stroke in order to assess: (1) the quality of the cohorts and the statistical methods used in their development; and (2) their external performance. We aimed to compare clinical prediction models with clinicians' informal predictions in a new prospective cohort study.

\section{Methods}

The analysis protocol is available at [5]. We searched Medline and EMBASE databases from 1980 to 19 April 2013 with an electronic search strategy using a search term for 'stroke' and synonyms for 'clinical prediction models' [see Additional file 1] [6,7]. We also searched reference lists, personal files and Google Scholar [8] for citations of relevant articles.

\section{Inclusion criteria}

Eligible articles developed and/or evaluated a multivariable clinical prediction model for the risk of recurrent ischemic stroke, myocardial infarction (MI) or all vaso-occlusive arterial events in cohorts of adult patients with ischemic stroke (or mixed cohorts of ischemic stroke and transient ischemic attack (TIA). We excluded any studies using cohorts that included hemorrhagic strokes. We made no language restrictions.

\section{Data extraction}

One author (DDT) screened all titles and abstracts identified by the electronic search against the inclusion criteria prior to full text assessment. Two authors (DDT and WNW) extracted data independently with a detailed data extraction form developed and piloted by three of the authors (DDT, GDM and WNW). We resolved discrepancies by discussion. We adapted quality items from similar systematic reviews [6,7,9-13] (Table 1) as no recommended tool for the appraisal of quality of prediction models currently exists. We distinguished two types of articles: (1) development studies reporting the construction of a prediction model, and (2) evaluation studies (also known as validation studies) assessing model performance in a cohort of new patients.

All measures of model performance were extracted along with any associated measures of uncertainty (for example, 95\% confidence intervals $(\mathrm{CI})$ or standard error). Two commonly used measures of performance are: 'calibration' and 'discrimination' [23]. Calibration summarizes how well the observed events match the predicted events by dividing the cohort into groups of predicted risk (for example, quintiles or deciles) and comparing the mean predicted risk with the observed frequency. Discrimination summarizes how well a model separates patients with the event in follow-up from those without. The $c$-statistic is a commonly used rank order measure of discrimination ranging from no better than chance (0.5) to perfect (1.0) discrimination. For a given pair of patients, one with the event of interest and one without, the $c$-statistic is interpreted as the probability that a greater predicted risk is given to the patient with the event than the patient without. In logistic regression the Area under the Receiver Operating Characteristic Curve (AUROCC) is equivalent to the $c$-statistic.

\section{Meta-analysis}

If three or more studies assessed a model's performance we performed a random-effects meta-analysis using the DerSimonian and Laird method [24] (implemented with the 'metafor' package [25] in $R$ version 2.13.1). A randomeffects meta-analysis allows for differences in model performance that may be explained by differing case mix between studies (for example, older patients or more severe baseline strokes and so on). We estimated the 95\% prediction interval (PI) associated with the individual pooled estimates which differs somewhat from the CI [26]. The CI summarizes the precision of a parameter estimate whereas the PI provides a plausible range within which an unknown estimate will be expected to lie in 95\% of future samples. We assessed publication bias with Contour-enhanced funnel plots [27]. The PRISMA checklist for our review is available as an online supplement [see Additional file 1].

\section{Evaluation cohort}

Evaluation in an external cohort is the most robust test of model performance and generalizability. The Edinburgh Stroke Study (ESS) was a prospective observational study of stroke patients admitted to the Western General Hospital in Edinburgh between April 2002 and May 2005 with a minimum follow-up of one year. Details on the study's design are available elsewhere [28]. Clinicians were asked to use 'gut-feeling' to estimate the absolute risk of a recurrent stroke or a vascular event (that is, stroke, MI or vascular death) within one year in patients seen as outpatients. We compared models we identified using measures of discrimination and calibration to clinicians' informal estimations.

\section{Results}

We screened 12,456 articles by title and abstract (PRISMA diagram Figure 1), thirteen of which were eligible for 
Table 1 Quality assessment of articles

\begin{tabular}{ll}
\hline $\begin{array}{l}\text { Quality item } \\
\text { Internal validity }\end{array}$ & Comment \\
$\begin{array}{l}\text { Sample cohort } \\
\text { Loss to follow up }\end{array}$ & $\begin{array}{l}\text { Prospectively collected data are of greater quality than retrospectively collected data and are preferred for model } \\
\text { development [14]. } \\
\text { Loss to follow up is common. Investigators should state the number of patients lost (or else the completeness } \\
\text { of follow-up [15] which takes into account the duration of follow-up) along with reasons/explanations. } \\
\text { An arbitrary proportion thought adequate for analysis is 90\% complete follow-up [7]. }\end{array}$ \\
$\begin{array}{l}\text { Predictive/outcome variables } \\
\text { Missing values }\end{array}$ & $\begin{array}{l}\text { A transparent summary of missing data and the methods used to handle them should be provided. Complete-case } \\
\text { analysis should be avoided in favor of multiple imputation methods [16,17]. A general rule of thumb suggests that } \\
\text { imputation should be considered if the proportion of missingness exceeds 5\% of the data [18]. }\end{array}$
\end{tabular}

Statistical validity

Model building strategy

Handling of continuous variables

sample size

Model evaluation

Evaluation

Description of external cohort

Discrimination and calibration
A priori clinical knowledge should be used to inform selection of risk factors. Data driven predictor selection (for example, stepwise selection) should be avoided where possible [19,20].

Arbitrary categorization should be avoided [21]. Defined cut-points must be based on clinical reasoning.

The sample size used in derivation (derivation sample) must be reported along with a sufficient description of baseline characteristics. The number of patients with the outcome event in follow-up (effective sample size) should be reported: 10 events per fitted parameter is often used as a minimum number [22].

Internal validation techniques (for example, bootstrap sampling or cross-validation) provide a minimum check of overfitting and optimism. External evaluation in new data is the most rigorous assessment of model generalizability.

A description of the baseline characteristics should be reported to enable a comparison of the validation cohort to the development cohort.

Discrimination metrics should be provided, for example, the area under the receiver operating characteristic curve (AUROCC). Model calibration should be studied using a calibration plot with estimated slope and intercept provided. review. A further ten were identified from reference list checks and forward citation searches in Google Scholar. We found twelve development studies [see Additional file 1 and Figure 2] that developed a total of 31 models (a median of two per study, interquartile range (IQR) one to three). We found eleven evaluation studies that evaluated four models [see Additional file 1]. Only one relevant study written in a language other than English was included [29].

\section{Model development studies: cohort characteristics}

Studies which collect data prospectively have a lower risk of information and selection biases for both baseline data and outcome events occurring during follow-up. Most studies used prospectively collected data, although four of twelve did not [30-33], one of which [33] used prospective trial data but included retrospective events obtained beyond the trial's original follow-up period. Few (four of twelve) development studies recruited patients consecutively from routine practice $[30,31,34,35]$. Loss of patients to follow-up often occurs when studies last for long time periods. Most (nine of twelve) [30-33,35-39] development studies reported loss to follow up; where this could be calculated (seven of eight) [31-33,35-39] rates of loss were small (less than 5\%).

The most frequent variables included in multivariable clinical prediction models were: age, history of TIA or stroke, history of hypertension, and diabetes [see Additional file 1]. Five articles $[31,32,34,36,39]$ defined all predictors, three $[30,35,37]$ defined only some, and four $[33,38,40,41]$ did not define any. Most articles defined outcome adequately, although three did not define the outcome and/or the duration of follow-up [38,40,41].

Missing baseline data occur frequently when collecting information from patients. A complete case analysis using only those patients with complete baseline data risks selection bias and loss of information. Five of the development studies [32-34,38,41] reported missing data, four [32-34,38] of which stated the impact a complete case analysis had on the derivation sample size. No attempts were made to impute missing data.

\section{Model development studies: statistical methods}

Most investigators collect more potential predictors than are included in a final model. Data dependent methods (for example, univariate selection or stepwise selection) are often used to select a few important variables from those available to develop a prediction model. This can lead to over-fitted models that perform over-optimistically in their development datasets which may be impossible to replicate in external evaluation [42]. Most of the studies used data dependent variable selection methods: stepwise selection (two of twelve) [32,35]; univariate significance tests (four of twelve) [30,31,34,36]; and further reduction 


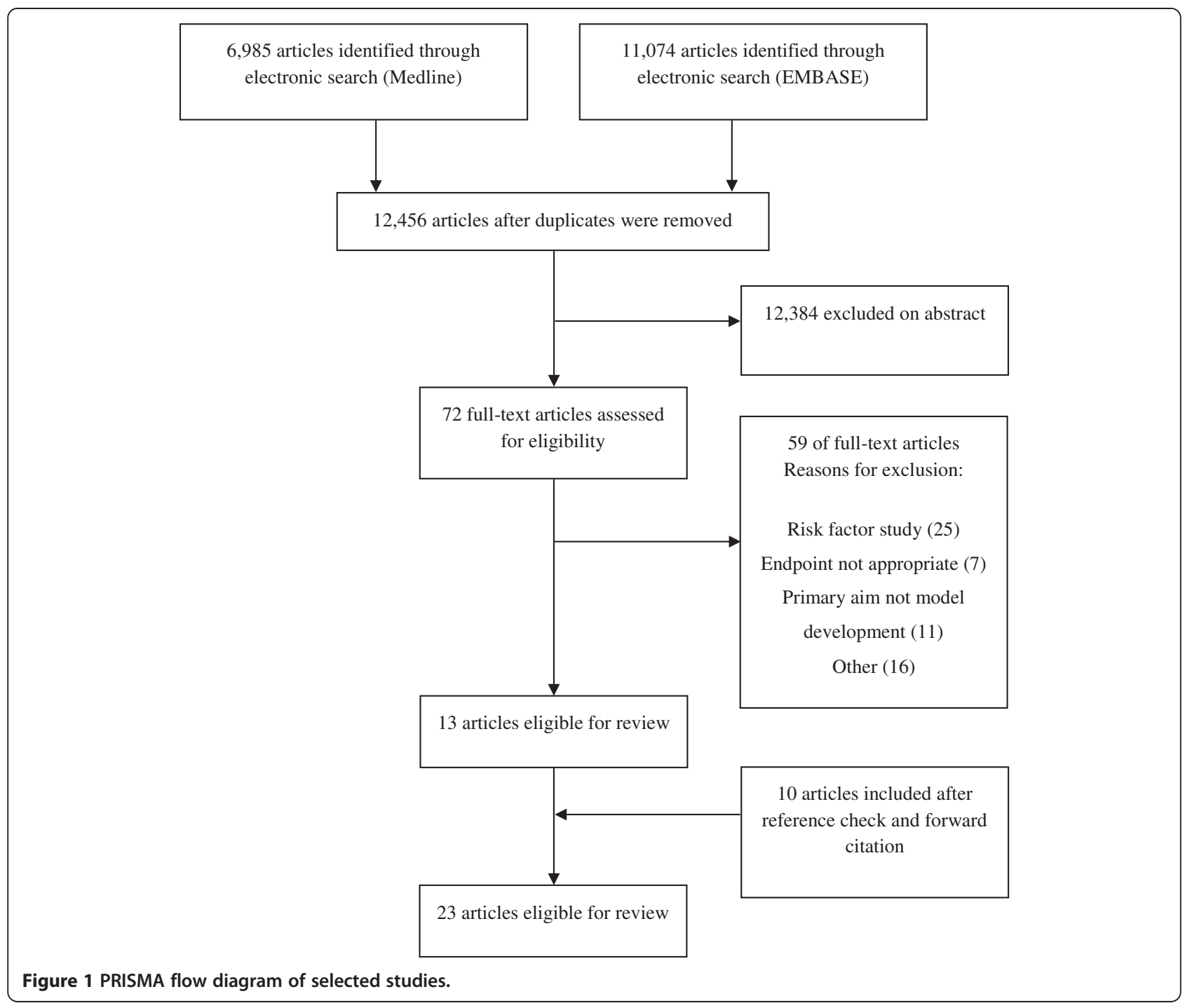

of univariate selection by inspection of multivariable significance (two of twelve) [33,38]. Three modifications of pre-existing prediction models were identified with new predictors chosen by clinical justification $[37,39,41]$. One study gave no description of how variables were selected [40].

Internal evaluation methods can use the model development data to provide optimism-corrected estimates of model performance. Few authors internally assessed the performance of their models (three of twelve) using such cross-validation methods [30,31,37].

Models were derived using Cox proportional hazard regression (nine of twelve) [30,32-36,38,39,41] or multivariable binary logistic regression (three of twelve) $[31,37,40]$. Most studies presented their models as point scores (seven of twelve) by rounding regression coefficients [30-32,34,37,40,41]. The categorization of a continuous predictor results in the loss of information. The majority of studies categorized continuous predictors (eight of twelve) [30-32,34,36,37,40,41], only one of which gave some clinical justification for the cut-points chosen [37]. The remaining four studies used a mixture of categorized and continuous variables $[33,35,38,39]$.

A common rule of thumb used in prediction model literature is the 'ten events per tested variable' (10 EPV) rule. The median total sample size across the twelve development studies was 1,132 (IQR 522 to 3,123). Where reported (nine of twelve), the median number of events was 73 (IQR 60 to 102). Only one of the five studies where the EPV could be calculated had more than the minimum recommended EPV [37].

\section{Model evaluation studies: study characteristics}

The ESSEN Stroke Risk Score (ESRS) [40], the Stroke Prognosis Instrument II (SPI-II) [41], the Recurrence Risk Estimator at 90 days (RRE-90) [30] and the Life Long 


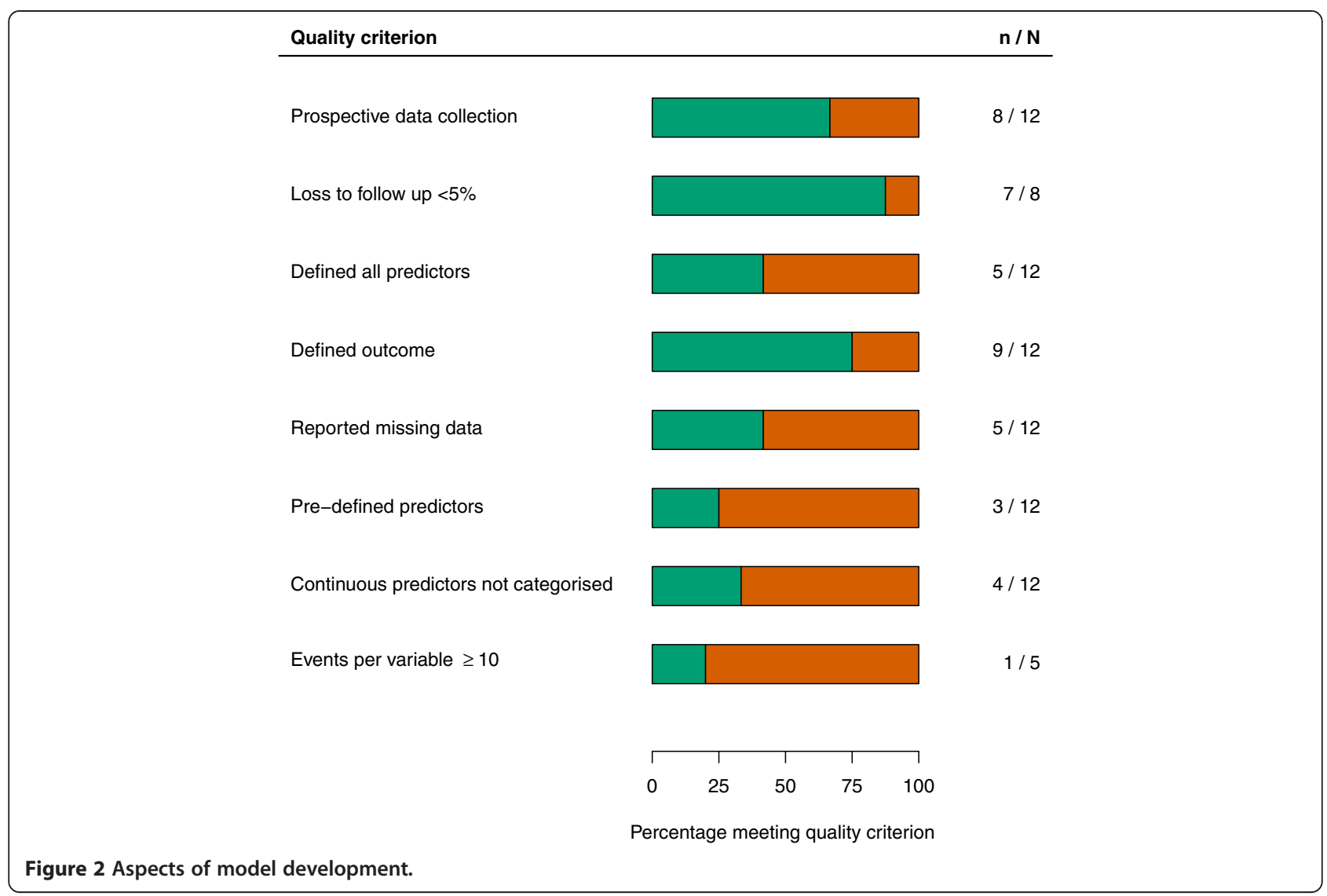

After Cerebral ischemia (LiLAC) [33] were externally evaluated in eleven different studies [see Additional file 1]. We identified four additional evaluations among the model development studies [30,37,39,41] giving fifteen evaluation cohorts: five evaluations of the ESRS [29,37,43-45]; three of the SPI-II [41,46,47]; five head-to-head comparisons of the ESRS and the SPI-II [30,39,48-51]; one head-to-head comparison of the ESRS and the RRE-90 [52]; and one comparing the ESRS, the SPI-II and the LiLAC models [50].

The median sample size in the 15 evaluation cohorts was 1,286 (IQR 619 to 5,004). Various combinations of events and follow-up periods were used yielding 49 specific AUROCC values for extraction [see Additional file 1]. Where the effective sample size could be determined the median size was 86 (IQR 58 to 134).

\section{Model evaluation studies: statistical performance}

The pooled AUROCC value for the ESRS was 0.60 (95\% CI 0.59 to 0.62 ) and for the SPI-II was 0.62 (95\% CI 0.60 to 0.64 ) (Figure 3). Six head-to-head comparisons of the ESRS and the SPI-II were identified. Four of these [39,49-51] (the other two [30,48] used much shorter follow-up periods) were pooled to calculate the AUROCC estimates: 0.61 (95\% CI 0.58 to 0.64$)$ with $95 \%$ PI $(0.29$ to 0.93 ) and 0.62 (95\% CI 0.59 to 0.66$)$ with $95 \%$ PI (0.23 to 0.99), respectively, for the ESRS and the SPI-II scores. These findings were robust to sensitivity analyses [see Additional file 1]. One evaluation study for the RRE-90 score estimated an AUROCC of 0.72 (95\% CI 0.64 to 0.80 ) [52] and another of the LiLAC score estimated an AUROCC of 0.65 (95\% CI 0.61 to 0.70) [50]. We identified two evaluations of the ABCD2 score $[48,52,53]$. Although the $\mathrm{ABCD} 2$ score was developed and designed for patients with TIA (and, therefore, did not meet our inclusion criteria) its performance was similar to other clinical prediction models for recurrent stroke (Figure 3). Only one study assessed the calibration of the SPI-II score which found it to be good but only after re-calibration [47]. There was no evidence for small study (that is, publication) bias [see Additional file 1].

\section{Model evaluation: comparative performance with clinical gestalt}

Baseline characteristics for the ESS can be found online [see Additional file 1].We were able to evaluate five of twelve models in the ESS (Table 2). In the ESS data, 575 patients had informal predictions for vascular outcomes by one year. We were able to obtain information regarding thirteen of the clinicians making predictions for 542 (94\%) of the patients. Of these: eight were neurologists (62\%) and five were stroke physicians (38\%); seven were in training (54\%) and six were fully trained (46\%). The 


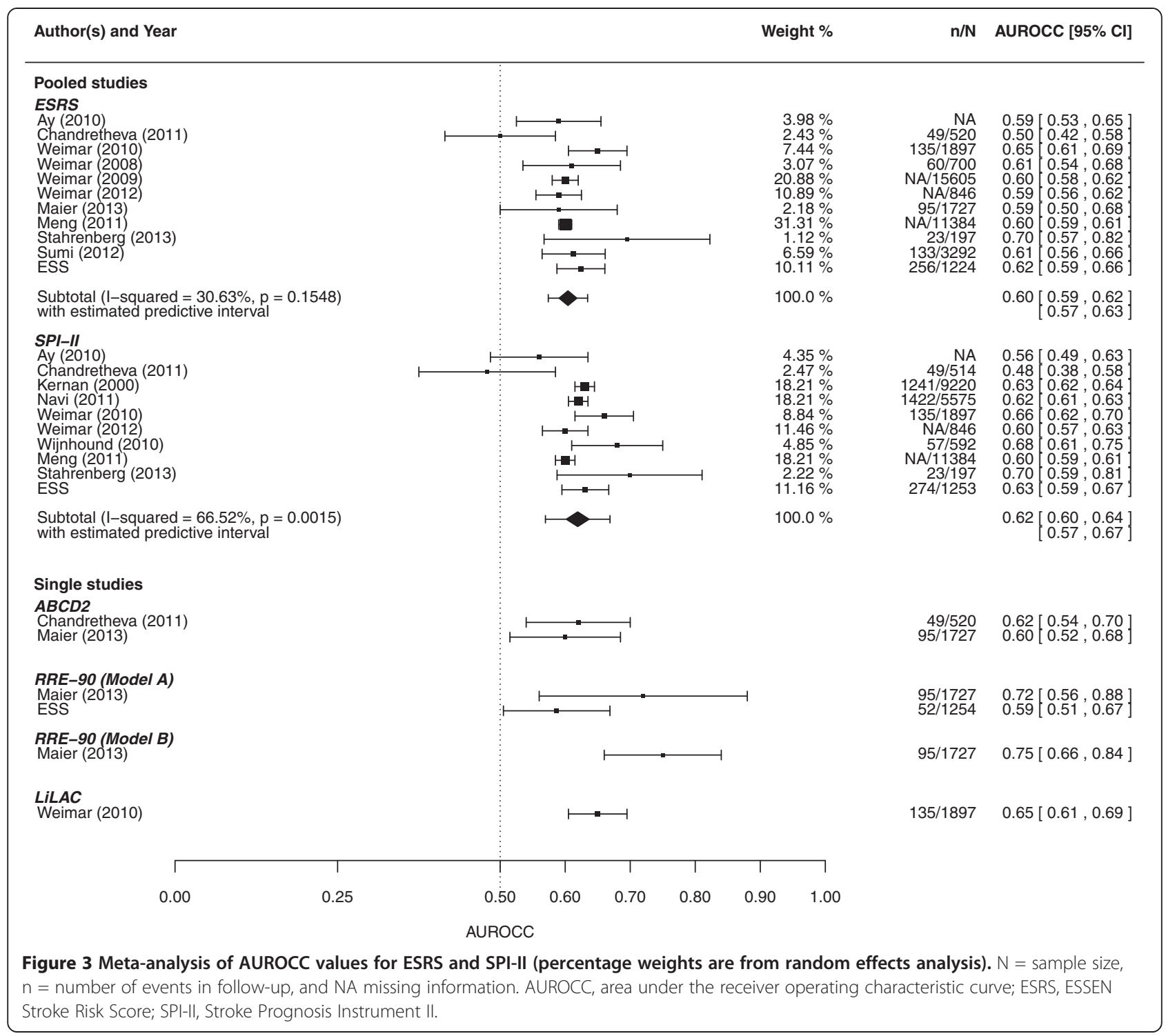

Table 2 Performance of models in the Edinburgh Stroke Study

\begin{tabular}{|c|c|c|c|c|c|c|c|c|c|}
\hline \multirow[b]{2}{*}{ Model } & \multicolumn{3}{|c|}{ Recurrent stroke $(50 / 671)^{a}$} & \multicolumn{3}{|c|}{ Any vascular event $(80 / 671)^{a}$} & \multicolumn{3}{|c|}{ Original development outcome ${ }^{\mathbf{b}}$} \\
\hline & $n / N$ & AUROCC & $95 \% \mathrm{Cl}$ & $\mathrm{n} / \mathrm{N}$ & AUROCC & $95 \% \mathrm{Cl}$ & $n / N$ & AUROCC & $95 \% \mathrm{Cl}$ \\
\hline Clinical gestalt & $40 / 575$ & 0.53 & 0.44 to 0.63 & $63 / 574$ & 0.56 & 0.48 to 0.64 & - & - & - \\
\hline ESRS & $50 / 664$ & 0.56 & 0.48 to 0.64 & $80 / 664$ & 0.57 & 0.50 to 0.63 & $101 / 1,224$ & 0.54 & 0.49 to 0.60 \\
\hline SPI-II & $50 / 669$ & 0.58 & 0.49 to 0.66 & $80 / 669$ & 0.59 & 0.52 to 0.66 & $274 / 1,253$ & 0.63 & 0.59 to 0.67 \\
\hline RRE-90 & $50 / 671$ & 0.61 & 0.52 to 0.69 & $80 / 671$ & 0.59 & 0.53 to 0.66 & $52 / 1,254$ & 0.59 & 0.51 to 0.67 \\
\hline Putaala & $50 / 669$ & 0.48 & 0.39 to 0.57 & $80 / 669$ & 0.56 & 0.49 to 0.63 & $269 / 1,247$ & 0.65 & 0.61 to 0.68 \\
\hline Dhamoon & $50 / 668$ & 0.60 & 0.52 to 0.68 & $80 / 668$ & 0.61 & 0.54 to 0.67 & $205 / 1,253$ & 0.73 & 0.69 to 0.76 \\
\hline
\end{tabular}

Cell entries are AUROCCs for a recurrent stroke by one year, all vascular events by one year, and the outcome as defined in development. Few patients were deleted due to missingness for prediction models ( 671 outpatients with 50 strokes in follow-up and 80 vascular events; and for all patients 1,257, 102 and 274 respectively). ${ }^{\mathrm{a}}$ Outpatients only; ${ }^{b}$ one year follow-up was available for all patients in the ESS; ' $M$ odel A was the clinical based model. AUROCC, area under the receiver operating characteristic curve; Cl, confidence interval; ESRS, ESSEN Stroke Risk Score; RRE-90, Recurrence Risk Estimator at 90 days; SPI-II, Stroke Prognosis Instrument II. 
median number of patients seen per clinician was seven (ranging from 1 to 217). For recurrent stroke within one year clinicians discriminated poorly between those who did and those who did not suffer an event with an AUROCC of 0.54 (95\%CI 0.44 to 0.62). Formal prediction also discriminated poorly with AUROCC measures varying between 0.48 and 0.61 . For risk of vascular events, clinicians again discriminated poorly with an AUROCC of 0.56 (95\%CI 0.48 to 0.64 ) and formal prediction ranged from 0.56 to 0.61 . The AUROCCs from the ESRS and the SPI-II were calculated for all patients in the ESS for any vascular event and added to the meta-analysis.

\section{Discussion}

We found four externally evaluated clinical prediction models for the prediction of recurrent stroke and MI after stroke: the ESRS, the SPI-II, the RRE-90 and LiLAC. The discriminative performances of the models were similar to one another, but only modest at best, with AUROCC values ranging from 0.60 to 0.72 . The performance of some of the clinical prediction models although modest was similar to experienced clinicians.

There were some weaknesses in the methodology of model development which may explain the modest performance observed in external evaluation studies of clinical prediction models. First, continuous variables were often categorized which leads to a loss of predictive information. Second, data-dependent variable selection may have led to over-fitting of models to the observed data. Third, cohorts were generally too small for reliable model development: we found only one study with more than the recommended $10 \mathrm{EPV}$. Small samples can lead to prediction models that are over-fit on the available data which is further compounded by implementing a complete case analysis. Fourth, the cohorts used to develop the models had weaknesses that are frequent in epidemiological studies: there were missing baseline data; whether the recruited patients were representative of those seen in routine clinical practice was uncertain; some data were collected retrospectively; and most cohorts did not record all potentially predictive variables. For example, the presence of multiple infarcts on MR scanning was only considered in one model $[30,54]$.

While it seems more likely that a well-developed model will have better performance in external evaluation, the only reliable method for choosing between models is their performance in evaluation studies of representative patients. Despite the differences in the methods of derivation of the ESRS, the SPI-II and the LiLAC, they discriminated similarly (and modestly) between patients with and without recurrent stroke [50]. The ESRS and the SPI-II have four predictors in common (age, history of TIA or stroke, diabetes and blood pressure).
Three head-to-head comparisons demonstrated a relative difference in AUROCC which did not exceed 2\% [49-51].

This is one of the few studies of the performance of clinicians' predicting vascular events. Although such investigations perhaps provide the most robust argument for or against the use of statistical prediction, they remain rare. For example, there are many prediction rules for poor outcome or disability after stroke [55] but few have been tested against clinicians' informal predictions [56].

\section{Implications for research}

Although discrimination of recurrent events by clinical prediction models was poor, our study indicates that it may be similar to informal clinicians' prediction. In addition, we identified a number of areas that could improve the discrimination of clinical prediction models for recurrent stroke or MI that future model developers could consider: (1) using all the available information from a cohort by avoiding the categorization of continuous predictors and using multiple imputation of missing data where a complete case analysis would exclude a significant proportion of the cohort; (2) reporting regression coefficients (that is, prior to any transformation) to allow more accurate evaluation of models in independent cohorts. Point score models are probably obsolete as more precise predictions can easily be obtained using applications accessed via mobile computers at the bedside. There are too many proposed models in clinical practice to remember them all, and it is only sensible that they should be available electronically; and finally, (3) measuring whether newly identified predictors (for example, blood markers or imaging techniques) add to the accurate classification of patients over more easily measured variables, for example using the net reclassification index [39,57].

A number of methodological decisions in model development may lead to clinical prediction models that make less accurate predictions [58] and we believe that an agreed set of guidelines in model development and reporting in healthcare would be helpful to developers and users of clinical prediction models alike [59].

\section{Limitations of the study}

Assessing the quality of studies of predictive models is difficult, and there is no widely agreed set of guidelines. This is likely to become an increasing problem as such studies are frequent and very likely will begin to influence practice. Our electronic search was overly sensitive and returned a small number of relevant articles; hence, we did not perform additional searches of the 'grey' literature. This is an unfortunate artefact of poor indexing, as there is no Medical Subject Heading (MESH) term for clinical prediction models. We attempted to work around these limitations with forward citation searching in Google Scholar. The ESS did not classify stroke according to the Causative 
Classification of Stroke System (CCS); we instead manipulated a record of classification as per the Trial of Org 10172 in Acute Stroke Treatment (TOAST) algorithm to a format that closely resembled the CCS.

\section{Conclusions}

We found that the available clinical prediction models for recurrent stroke and MI after stroke discriminated modestly between patients who do and do not have recurrent events. Clinicians' informal predictions discriminated similarly to the models. Aspect of study design and statistical methodology were poor amongst model development studies, however, and performance might be improved with better methods.

\section{Additional file}

\section{Additional file 1: Electronic search term implemented in Medline} and EMBASE. Further detail of included studies.

\section{Abbreviations \\ AUROCC: area under the receiver operating characteristic curve; Cl: confidence interval; EPV: events per variable; ESRS: ESSEN Stroke Risk Score; ESS: The Edinburgh Stroke Study; IQR: interquartile range; Ml: myocardial infarction; PI: prediction interval; RRE-90: Recurrence Risk Estimator at 90 days; SPI-II: Stroke Prognosis Instrument II; TIA: transient ischemic attack.}

\section{Competing interests}

The authors declare that they have no competing interests.

\section{Authors' contributions}

WNW conceived the study. WNW, GDM and DDT contributed to the study design. WNW and DDT collected data. DDT carried out the analyses and drafted the first manuscript. WNW, MD, CLMS and GDM revised the manuscript. This study was supervised by GDM and WNW. CLMS was the principal investigator of the ESS. All authors read and approved the final manuscript.

\section{Acknowledgements}

We would like to thank Marshall Dozier for her help in validating our electronic search string, Cristina Matthews for translating a non-English article, Professor Joanna Wardlaw for her helpful comments on an earlier draft, and Aidan Hutchison for his extraction of data from the ESS database.

\section{Financial disclosure}

This study received no additional funding. DDT is supported by an MRC HTMR grant (G0800803). WNW is supported by an MRC Clinician Scientist Fellowship G0902303.

\section{Author details}

'Edinburgh MRC Hub for Trials Methodology Research, Centre for Population Health Sciences, University of Edinburgh Medical School, Teviot Place, Edinburgh EH8 9AG, UK. ${ }^{2}$ Division of Clinical Neurosciences, University of Edinburgh, Bramwell Dott Building, Western General Hospital, Edinburgh EH4 2XU, UK.

Received: 21 November 2013 Accepted: 28 February 2014 Published: 4 April 2014

\section{References}

1. Mohan KM, Wolfe CD, Rudd AG, Heuschmann PU, Kolominsky-Rabas PL, Grieve AP: Risk and cumulative risk of stroke recurrence. Stroke 2011 42:1489-1494
2. Woodward M, Brindle P, Tunstall-Pedoe H: Adding social deprivation and family history to cardiovascular risk assessment: the ASSIGN score from the Scottish Heart Health Extended Cohort (SHHEC). Heart 2007, 93:172-176.

3. Anderson KM, Odell PM, Wilson PW, Kannel WB: Cardiovascular disease risk profiles. Am Heart J 1991, 121:293-298.

4. Gage BF, Waterman AD, Shannon W, Boechler M, Rich MW, Radford MJ: Validation of clinical classification schemes for predicting stroke: Results from the national registry of atrial fibrillation. JAMA 2001, 285:2864-2870.

5. Whiteley W, Murray G, Thompson D: The performance of multivariate prognostic models to predict occlusive and haemorrhagic vascular events after stroke; a systematic review. Available at: http://www.dcn.ed. ac.uk/dcn/documents/profile_protocols/whiteley_p2.pdf.

6. Collins GS, Mallett S, Omar O, Yu LM: Developing risk prediction models for type 2 diabetes: a systematic review of methodology and reporting. BMC Med 2011, 9:103.

7. Counsell C, Dennis M: Systematic review of prognostic models in patients with acute stroke. Cerebrovasc Dis 2001, 12:159-170.

8. Google Scholar. http://scholar.google.co.uk/.

9. Hayden JA, Côté P, Bombardier C: Evaluation of the quality of prognosis studies in systematic reviews. Ann Intern Med 2006, 144:427-437.

10. Mallett $S$, Royston $P$, Dutton $S$, Waters $R$, Altman D: Reporting methods in studies developing prognostic models in cancer: a review. BMC Med 2010, 8:20.

11. Mushkudiani NA, Hukkelhoven CW, Hernández AV, Murray GD, Choi SC, Maas Al, Steyerberg EW: A systematic review finds methodological improvements necessary for prognostic models in determining traumatic brain injury outcomes. J Clin Epidemiol 2008, 61:331-343.

12. Kwakkel G, Wagenaar RC, Kollen BJ, Lankhorst GJ: Predicting disability in stroke-a critical review of the literature. Age Ageing 1996, 1996:479-489.

13. Laupacis A, Sekar N, Stiell LG: Clinical prediction rules. JAMA 1997, 277:488-494.

14. Moons KG, Royston P, Vergouwe Y, Grobbee DE, Altman DG: Prognosis and prognostic research: what, why, and how? BMJ 2009, 338:b375.

15. Clark TG, Altman DG, Stavola BL: Quantification of the completeness of follow-up. Lancet 2002, 359:1309-1310.

16. Rubin DB: Multiple imputation after 18+ years. J Am Stat Assoc 1996, 91:473-489.

17. Vergouwe Y, Royston P, Moons KG, Altman DG: Development and validation of a prediction model with missing predictor data: a practical approach. J Clin Epidemiol 2010, 63:205-214.

18. Harrell FE: Regression Modeling Strategies: With Applications to Linear Models, Logistic Regression, and Survival Analysis. Springer; 2001.

19. Sun GW, Shook TL, Kay GL: Inappropriate use of bivariable analysis to screen risk factors for use in multivariable analysis. J Clin Epidemiol 1996, 49:907-916.

20. Steyerberg EW, Eijkemans MJ, Habbema JD: Stepwise selection in small data sets: a simulation study of bias in logistic regression analysis. J Clin Epidemiol 1999, 52:935-942.

21. Altman DG: Problems in dichotomizing continuous variables. Am J Epidemiol 1994, 139:442-445.

22. Peduzzi P, Concato J, Kemper E, Holford TR, Feinstein AR: A simulation study of the number of events per variable in logistic regression analysis. J Clin Epidemiol 1996, 49:1373-1379.

23. Steyerberg EW, Vickers AJ, Cook NR, Gerds T, Gonen M, Obuchowski N, Pencina MJ, Kattan MW: Assessing the performance of prediction models: a framework for traditional and novel measures. Epidemiology 2010, 21:128-138

24. DerSimonian R, Laird N: Meta-analysis in clinical trials. Control Clin Trials 1986, 7:177-188.

25. Viechtbauer $\mathrm{W}$ : Conducting meta-analyses in $\mathrm{R}$ with the metafor package. J Stat Softw 2010, 36:1-48.

26. Riley RD, Higgins JPT, Deeks Jj: Interpretation of random effects meta-analyses. BMJ 2011, 342:d549.

27. Peters JL, Sutton AJ, Jones DR, Abrams KR, Rushton L: Contour-enhanced meta-analysis funnel plots help distinguish publication bias from other causes of asymmetry. J Clin Epidemiol 2008, 61:991-996.

28. Whiteley W, Jackson C, Lewis S, Lowe G, Rumley A, Sandercock P, Wardlaw J, Dennis M, Sudlow C: Inflammatory markers and poor outcome after stroke: a prospective cohort study and systematic review of interleukin-6. PLoS Med 2009, 6:e1000145. 
29. Alvarez-Sabin J, Quintana M, Rodriguez M, Arboix A, Ramirez JM, Fuentes B: Validation of the Essen risk scale and its adaptation to the Spanish population, Modified Essen risk scale. Neurologia 2008, 23:209-214.

30. Ay H, Gungor L, Arsava EM, Rosand J, Vangel M, Benner T, Schwamm LH, Furie KL, Koroshetz WJ, Sorensen AG: A score to predict early risk of recurrence after ischemic stroke. Neurology 2010, 74:128-135.

31. Kamouchi M, Kumagai N, Okada Y, Origasa H, Yamaguchi T, Kitazono T: Risk score for predicting recurrence in patients with ischemic stroke: the Fukuoka Stroke Risk Score for Japanese. Cerebrovasc Dis 2012, 34:351-357.

32. Kernan WN, Horwitz RI, Brass LM, Viscoli CM, Taylor KJ: A prognostic system for transient ischemia or minor stroke. Ann Intern Med 1991, 114:552-557.

33. van Wijk I, Kappelle LJ, van Gijn J, Koudstaal PJ, Franke CL, Vermeulen M, Gorter JW, Algra A: Long-term survival and vascular event risk after transient ischaemic attack or minor ischaemic stroke: a cohort study. Lancet 2005, 365:2098-2104.

34. Pezzini A, Grassi M, Del Zotto E, Lodigiani C, Ferrazzi P, Spalloni A, Patella R Giossi A, Volonghi I, lacoviello L, Magoni M, Rota LL, Rasura M, Padovani A: Common genetic markers and prediction of recurrent events after ischemic stroke in young adults. Neurology 2009, 73:717-723.

35. Putaala J, Haapaniemi E, Metso AJ, Metso TM, Artto V, Kaste M, Tatlisumak T: Recurrent ischemic events in young adults after first-ever ischemic stroke. Ann Neurol 2010, 68:661-671.

36. Dhamoon MS, Tai W, Boden-Albala B, Rundek T, Paik MC, Sacco RL, Elkind MS: Risk of myocardial infarction or vascular death after first ischemic stroke: the Northern Manhattan Study. Stroke 2007, 38:1752-1758.

37. Sumi S, Origasa H, Houkin K, Terayama Y, Uchiyama S, Daida H, Shigematsu H, Goto S, Tanaka K, Miyamoto S, Minematsu K, Matsumoto M, Okada Y, Sato M, Suzuki N: A modified Essen stroke risk score for predicting recurrent cardiovascular events: development and validation. Int J Stroke 2013, 8:251-257.

38. Suzuki N, Sato M, Houkin K, Terayama Y, Uchiyama S, Daida H, Shigematsu H, Goto S, Tanaka K, Origasa H, Miyamoto S, Minematsu K, Matsumoto M, Okada Y: One-year atherothrombotic vascular events rates in outpatients with recent non-cardioembolic ischemic stroke: the EVEREST (Effective Vascular Event REduction after STroke) Registry. J Stroke Cerebrovasc Dis 2012, 21:245-253.

39. Stahrenberg R, Niehaus CF, Edelmann F, Mende M, Wohlfahrt J, Wasser K, Seegers J, Hasenfuß G, Gröschel K, Wachter R: High-sensitivity troponin assay improves prediction of cardiovascular risk in patients with cerebral ischaemia. J Neurol Neurosurg Psychiatry 2013, 84:479-487.

40. Diener HC, Ringleb PA, Savi P: Clopidogrel for the secondary prevention of stroke. Expert Opin Pharmacother 2005, 6:755-764.

41. Kernan WN, Viscoli CM, Brass LM, Makuch RW, Sarrel PM, Roberts RS, Gent M, Rothwell P, Sacco RL, Liu RC, Boden-Albala B, Horwitz Rl: The stroke prognosis instrument II (SPI-II): a clinical prediction instrument for patients with transient ischemia and nondisabling ischemic stroke. Stroke 2000, 31:456-462.

42. Steyerberg EW: Clinical Prediction Models: A Practical Approach to Development, Validation, and Updating. New York: Springer; 2009.

43. Fitzek S, Leistritz L, Witte OW, Heuschmann PU, Fitzek C: The Essen Stroke Risk Score in one-year follow-up acute ischemic stroke patients. Cerebrovasc Dis 2011, 31:400-407.

44. Weimar C, Diener HC, Alberts MJ, Steg PG, Bhatt DL, Wilson PW, Mas JL, Röther J, REduction of Atherothrombosis for Continued Health Registry Investigators: The Essen Stroke Risk Score predicts recurrent cardiovascular events. Stroke 2009, 40:350-354.

45. Weimar C, Goertler M, Rother J, Ringelstein EB, Darius H, Nabavi DG, Kim IH, Benemann J, Diener HC, SCALA Study Group: Predictive value of the Essen Stroke Risk Score and Ankle Brachial Index in acute ischaemic stroke patients from 85 German stroke units. J Neurol Neurosurg Psychiatry 2008, 79:1339-1343

46. Navi BB, Kamel H, Sidney S, Klingman JG, Nguyen-Huynh MN, Johnston SC: Validation of the Stroke Prognostic Instrument-II in a large, modern, community-based cohort of ischemic stroke survivors. Stroke 2011 42:3392-3396

47. Wijnhoud AD, Maasland L, Lingsma HF, Steyerberg EW, Koudstaal PJ, Dippel DW: Prediction of major vascular events in patients with transient ischemic attack or ischemic stroke. Stroke 2010, 41:2178-2185.

48. Chandratheva A, Geraghty OC, Rothwell PM: Poor performance of current prognostic scores for early risk of recurrence after minor stroke. Stroke 2011, 42:632-637.
49. Meng $X$, Wang $Y$, Zhao $X$, Wang $C$, Li H, Liu L, Zhou Y, Xu J, Wang Y: Validation of the Essen Stroke Risk Score and the Stroke Prognosis Instrument II in Chinese patients. Stroke 2011, 42:3619-3620.

50. Weimar C, Benemann J, Michalski D, Muller M, Luckner K, Katsarava Z, Weber R, Diener HC, German Stroke Study Collaboration: Prediction of recurrent stroke and vascular death in patients with transient ischemic attack or nondisabling stroke: a prospective comparison of validated prognostic scores. Stroke 2010, 41:487-493.

51. Weimar C, Siebler M, Brandt T, Römer D, Rosin L, Bramlage P, Sander D: Vascular risk prediction in ischemic stroke patients undergoing in-patient rehabilitation - insights from the investigation of patients with ischemic stroke in neurologic rehabilitation (INSIGHT) registry. Int J Stroke 2013, 8:503-509.

52. Maier IL, Bauerle M, Kermer P, Helms HJ, Buettner T: Risk prediction of very early recurrence, death and progression after acute ischaemic stroke. Eur J Neurol 2013, 20:599-604.

53. Johnston SC, Rothwell PM, Nguyen-Huynh MN, Giles MF, Elkins JS, Bernstein $\mathrm{AL}$, Sidney $\mathrm{S}$ : Validation and refinement of scores to predict very early stroke risk after transient ischaemic attack. Lancet 2007, 369:283-292.

54. Kurth T, Stapf C: Here comes the sun? Neurology 2010, 74:102-103.

55. Veerbeek JM, Kwakkel G, van Wegen EE, Ket JC, Heymans MW: Early prediction of outcome of activities of daily living after stroke: a systematic review. Stroke 2011, 42:1482-1488.

56. Counsell C, Dennis M, McDowall M: Predicting functional outcome in acute stroke: comparison of a simple six variable model with other predictive systems and informal clinical prediction. I Neurol Neurosurg Psychiatry 2004, 75:401-405.

57. Pencina MJ, D'Agostino RB, Vasan RS: Evaluating the added predictive ability of a new marker: from area under the ROC curve to reclassification and beyond. Stat Med 2008, 27:157-172.

58. Bouwmeester W, Zuithoff NP, Mallett S, Geerlings MI, Vergouwe Y, Steyerberg EW, Altman DG, Moons KG: Reporting and methods in clinical prediction research: a systematic review. PLoS Med 2012, 9:e1001221.

59. Collins G: Opening up multivariable prediction models: consensus-based guidelines for transparent reporting. In BMJ; 2011

doi:10.1186/1741-7015-12-58

Cite this article as: Thompson et al:: Formal and informal prediction of recurrent stroke and myocardial infarction after stroke: a systematic review and evaluation of clinical prediction models in a new cohort. BMC Medicine 2014 12:58.

\section{Submit your next manuscript to BioMed Central and take full advantage of:}

- Convenient online submission

- Thorough peer review

- No space constraints or color figure charges

- Immediate publication on acceptance

- Inclusion in PubMed, CAS, Scopus and Google Scholar

- Research which is freely available for redistribution 